\title{
Femtosecond coherent seeding of a broadband Tm:fiber amplifier by an Er:fiber system
}

\author{
Sören Kumkar, ${ }^{1}$ Günther Krauss, ${ }^{1}$ Marcel Wunram, ${ }^{1}$ David Fehrenbacher, ${ }^{1}$ Umit Demirbas, ${ }^{1}$ \\ Daniele Brida, ${ }^{1,2}$ and Alfred Leitenstorfer ${ }^{1, *}$ \\ ${ }^{1}$ Department of Physics and Center for Applied Photonics, University of Konstanz, D-78457 Konstanz, Germany \\ ${ }^{2}$ IFN-CNR, Dipartimento di Fisica, Politecnico di Milano, Piazza Leonardo da Vinci 32, 20133 Milano, Italy \\ *Corresponding author: alfred.leitenstorfer@uni-konstanz.de
}

Received November 9, 2011; revised December 23, 2011; accepted December 23, 2011; posted December 23, 2011 (Doc. ID 157917); published February 8, 2012

\begin{abstract}
We generate broadband pulses covering the Yb: and Tm:silica amplification ranges with a passively phase-locked front end based on Er:fiber technology. Full spectral coherence of the octave-spanning output from highly nonlinear germanosilicate bulk fibers is demonstrated. Seeding of a high-power Tm:fiber generates pulses with a clean spectral shape and a bandwidth of $50 \mathrm{~nm}$ at a center wavelength of $1.95 \mu \mathrm{m}$, pulse energy of $250 \mathrm{~nJ}$, and repetition rate of $10 \mathrm{MHz}$. (c) 2012 Optical Society of America

OCIS codes: $320.7160,140.3510,190.7110$.
\end{abstract}

Applications of ultrafast pulsed radiation are increasingly based on fiber laser technology due to its inherent advantages such as compactness, stability, high power, and turn-key operation. Examples include materials processing [1], nonlinear microscopy [2], and precision metrology [3] . A new frontier for femtosecond fiber systems is represented by broadband seeding of various high-power amplification schemes. Owing to their extremely high efficiencies, $\mathrm{Tm}$ - and $\mathrm{Yb}$-doped systems are favorable for this task [4-6].

Recently, Tm:silica fiber amplifiers are attracting a lot of attention due to their broad gain bandwidth spanning from 1.85 to $2.1 \mu \mathrm{m}$ [7]. The long wavelength ensures a relatively high threshold for four-wave-mixing and stimulated Raman processes. Simultaneously, it supports single-mode operation at large mode field diameters [8], thus enabling high peak powers. Up to now, exploiting the full amplification bandwidth of Tm:fiber amplifiers has been limited by a lack of ultrabroadband and coherent seed sources. Only recently, a Tm:fiber oscillator has been introduced, delivering pulses with a duration of $78 \mathrm{fs}$ and a spectral width of $130 \mathrm{~nm}$ [9]. Other approaches based on well-established Er:fiber technology may be even more desirable. However, Raman self frequency shifting [10] or supercontinuum (SC) generation in microstructured fibers [11] turned out to be ineffective, since they provide seed pulses flawed by a significant degree of incoherence $[\underline{3}, \underline{12}]$.

In this Letter, we present a passively phase-locked and ultrabroadband source that is suitable for coherent seeding of both Yb: and Tm:fiber systems. This setup exploits Er:fiber technology combined with frequency conversion in highly nonlinear bulk fibers (HNF). It is worth noting that our approach allows phase coherent operation of synchronized multibranch systems at different amplification wavelengths, thus enabling synthesis of intense pulses down to single-cycle durations [13].

Figure 1(a) shows the schematic setup of the system. The seed source starts with a solitonic Er:fiber oscillator modelocked via a saturable absorber mirror. It is fol- lowed by a passively carrier-envelope phase-locked amplifier system. The output pulses, centered at $1.55 \mu \mathrm{m}$ with energies up to $8 \mathrm{~nJ}$ at a repetition rate of $40 \mathrm{MHz}$, are available at up to six distinct branches [14]. The key point of our system is the generation of ultrabroadband seed pulses in a highly nonlinear bulk germanosilicate fiber that we demonstrate to exhibit full spectral coherence. In detail, the $8 \mathrm{~nJ}$ output of the Er:fiber system is compressed in a silicon $(\mathrm{Si})$ prism sequence and then coupled into a combination of $9 \mathrm{~cm}$ polarization maintaining single-mode fiber (PM-SMF) followed by an $8 \mathrm{~mm}$ long germanosilicate HNF [15]. The variable insertion of the Si prism in the compressor stage allows fine control of the spectral phase of the driving pulse before propagating in the HNF assembly. In this way, we generate tunable spectra spanning from $800 \mathrm{~nm}$ to $2300 \mathrm{~nm}$. According to our simulations, the pulse first undergoes solitonic compression down to less than $20 \mathrm{fs}$ in the standard fiber section. Subsequently, it splits up in the HNF, thus leading to the formation of a red-shifting soliton and a dispersive wave at higher frequencies $[15,16]$. In Fig. 2(a), the solitonic part acquired with an extended InGaAs optical multichannel analyzer (OMA) is depicted (dashed lines). It is characterized by a full width at half maximum (FWHM) bandwidth of $350 \mathrm{~nm}$ centered at a wavelength of $1.94 \mu \mathrm{m}$. These parameters correspond to a transform-limited pulse duration of less than $20 \mathrm{fs}$. The spectrum covers the entire amplification range of Tm:silica. Figure 2(b) shows the corresponding dispersive wave acquired with an InGaAs-based optical spectrum analyzer (OSA). This component also exhibits a bandwidth larger than $300 \mathrm{~nm}$, including the wavelength range suitable for amplification with Yb:fibers around $1 \mu \mathrm{m}$.

To study the coherence properties of the spectrum after the HNF, we implement the interferometer depicted in Fig. 1(a) $[17,18]$. Two parallel outputs of our phaselocked system are amplified independently, compressed, and then used to pump two identical HNF stages providing the broadband seed pulses. In this way, we make sure 
that the coherence properties of both branches evolve separately from each other. Technical noise, which might lead to a loss of spectral coherence via amplitude-tophase conversion in the HNFs, is dominated by the amplifier stages, which are implemented independently. Also, any parametric amplification of quantum noise present in the HNFs will not be correlated in our parallel scheme. The output spectra of both branches are depicted as red and blue dashed lines in Figs. 2(a) and (b), respectively. These results demonstrate the excellent reproducibility of wavelength shifting over the entire range accessible with our HNFs. We then superimpose the two pulses with a 50/50 beam splitter and couple them into a PM-SMF to ensure perfect spatial overlap. A polarizer in front of the mode-matching fiber adjusts the correct polarization. A variable neutral density filter placed in one arm allows for fine balancing of the relative intensities. Temporal overlap is controlled via a variable delay line. The spectral interference that arises when the two pulses are slightly delayed with respect to each other represents the first-order coherence function [9]. It is depicted in Fig. 2 for both the solitonic (a) and the dispersive (b) part, respectively (black solid lines). We find an astonishing modulation depth of the interference fringes larger than $98 \%$ in the long wavelength region and above $99.5 \%$ in the shortwave part. This result shows the excellent degree of spectral coherence of both the SC and the distinct nonlinear Er:amplifiers used to generate the driving pulses for the HNFs. Note that the fringe visibility in the solitonic part is limited by the finite spectral resolution of the OMA of approximately $1 \mathrm{~nm}$. The remaining $0.5 \%$ residual of the modulation depth observed in the short wavelength region (where we have a resolution of $0.06 \mathrm{~nm}$ )

(a)

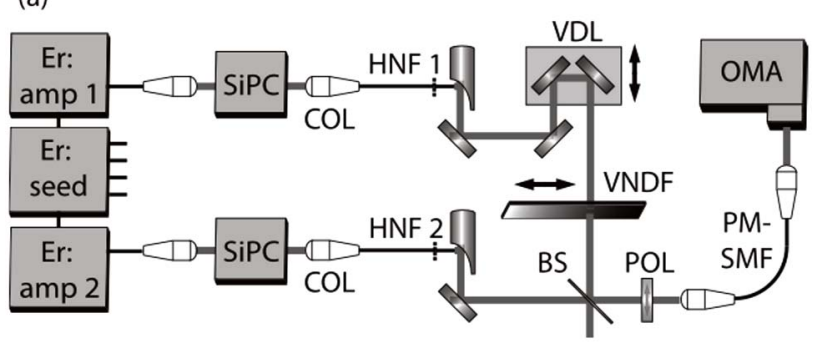

(b)

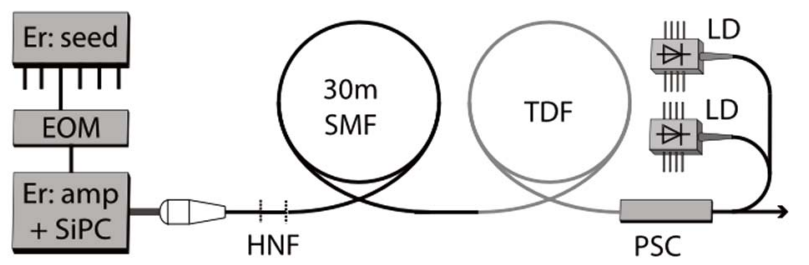

Fig. 1. (a) Schematic setup for the measurement of the coherence. Er:seed: passively phase stable seed source; Er:amp 1,2: fs Er:doped fiber amplifiers; SiPC: silicon prism compressors; HNF 1,2: highly nonlinear fiber assemblies; VDL: variable delay line; VNDF: variable neutral density filter; BS: beam splitter (50/50); POL: polarizer; PM-SMF: polarization maintaining single-mode fiber; OMA: optical multichannel analyzer. (b) Schematic setup of the Tm:amplifier. EOM: electro-optic modulator; SMF: single-mode fiber for pulse stretching; TDF: thulium-doped doubleclad fiber; PSC: pump-signal combiner; LD: multimode laser diodes emitting at $793 \mathrm{~nm}$.
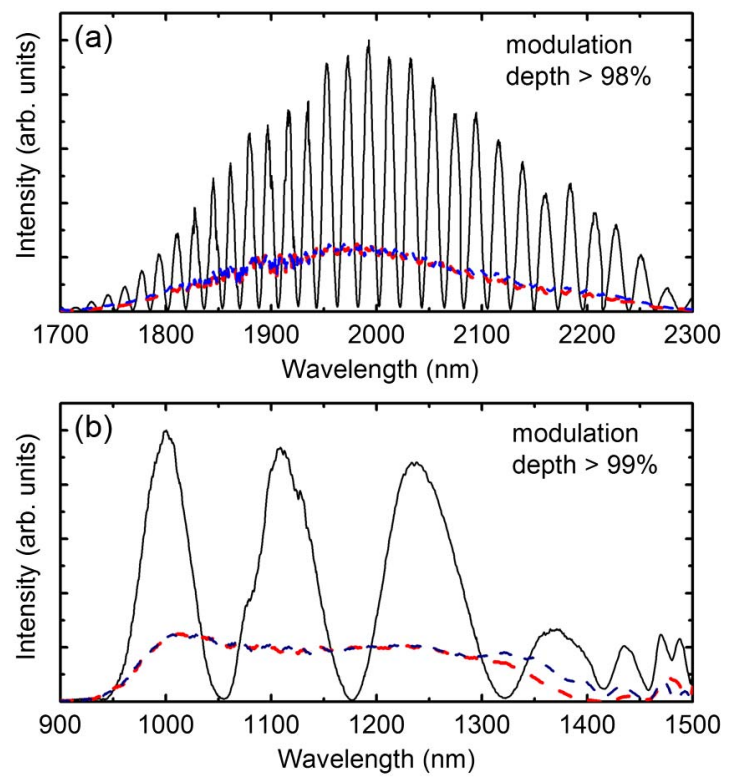

Fig. 2. (Color online) Spectra generated independently in the two parallel Er:amplifiers and highly nonlinear fibers (blue and red, dashed lines) and interference patterns after superposition (black lines). The solitonic part of the spectrum acquired with an OMA is plotted in (a) and the dispersive part in (b). Almost perfect contrast of the interference fringes demonstrates excellent coherence of the broadening process.

may be dominated by timing jitter between the two interferometer arms with a separated path length of $5 \mathrm{~m}$.

These results are very encouraging, since a significant loss of coherence has been reported recently for a similar setup, especially in the longwave solitonic part of an SC generated in a microstructured fiber pumped with an Yb:system [3]. The explanation for the superior coherence performance of our approach is as follows: the HNFs are cut to be shorter than the characteristic fission length, $L_{\text {fiss }}$, of the soliton. In this situation, the initial broadening process of the spectrum can reach its maximum without being affected by noise amplification due to modulation instabilities. The large bandwidth obtained already during the first fission period is a result of careful optimization of the dispersion of the bulk HNF, which for a pump wavelength of $1.55 \mu \mathrm{m}$ may be adjusted via both waveguide effects and Ge doping. In this way, we are able to fulfill the conditions suggested previously for a highly coherent and stable SC $[\underline{11}, \underline{19}]$. In particular, after the Si prism sequence, we measure a pulse duration of $120 \mathrm{fs}$ and a peak power of $25 \mathrm{~kW}$. These values correspond to an injected soliton order of $N=4.85$ and $L_{\text {fiss }}=15 \mathrm{~cm}$ inside the PMSMF [8]. The group velocity dispersion of this fiber is $\beta_{2}=1 \overline{9} .7 \mathrm{ps}^{2} / \mathrm{km}$, and the nonlinear coefficient amounts to $\gamma=1.3(\mathrm{~km} \cdot \mathrm{W})^{-1}$. The length is limited to $9 \mathrm{~cm}$ leading to a highly coherent solitonic precompression. According to our simulations, a pulse of a duration below $20 \mathrm{fs}$ and a peak power of $200 \mathrm{~kW}$ is then injected into the HNF [15]. This process results in a soliton order of $N=15$. With $\beta_{2}=-3.15 \mathrm{ps}^{2} / \mathrm{km}$ and $\gamma=9(\mathrm{~km} \cdot \mathrm{W})^{-1}$, we calculate $L_{\text {fiss }}=8.3 \mathrm{~mm}$, which matches the physical length of the HNF section.

The fractional Raman contribution to the overall nonlinearity $f_{R}$ also plays an important role for coherence 


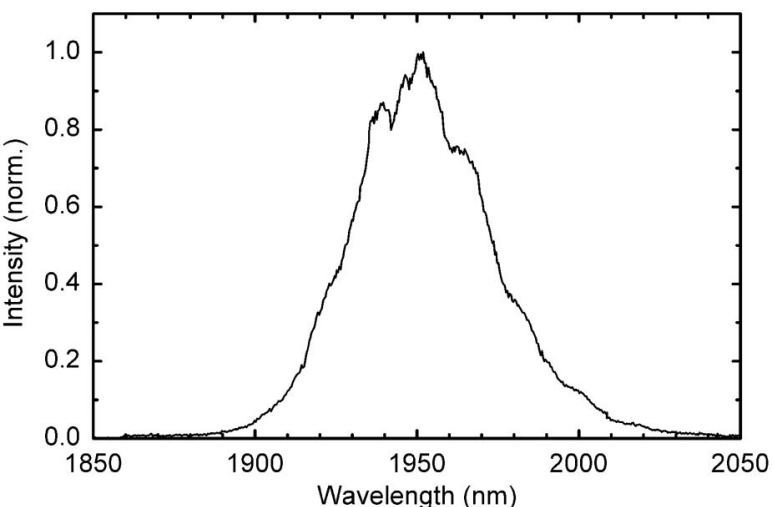

Fig. 3. Spectrum of the broadband output from the Tm:amplifier. The pulse energy amounts to $250 \mathrm{~nJ}$ at a repetition rate of $10 \mathrm{MHz}$.

properties of SC generation [3] . Because of the strong Ge doping of the bulk HNF, it should be significantly larger in our study as compared to pure fused silica $\left(f_{R}=0.18\right)$. This fact might also contribute to the excellent spectral coherence we observe.

To prove the capabilities of our laser source to effectively seed a high-power amplifier, we build the Tm:fiber system that is sketched in Fig. 1(b). Our scheme relies on purely single-mode cores with a diameter of $10 \mu \mathrm{m}$ ensuring diffraction-limited output and linear amplification for the generation of high-energy pulses. We reduce the repetition rate to $10 \mathrm{MHz}$ via an electro-optic modulator and stretch the temporal duration of the seed to $60 \mathrm{ps}$ by propagation in an SMF with a length of $30 \mathrm{~m}$, which is directly spliced to the output of the HNF. The amplification occurs in a doubleclad $\mathrm{Tm}^{3+}$ :fiber. It is pumped in counterpropagating direction by two multimode laser diodes at a wavelength of $793 \mathrm{~nm}$ that are coupled in via a fiber-based pump-signal combiner.

At the maximum pump power of $9 \mathrm{~W}$, we measure an average output power of $2.5 \mathrm{~W}$, corresponding to a pulse energy of $250 \mathrm{~nJ}$. To the best of our knowledge, this is the highest pulse energy reported from a broadband Tm:fiber amplifier to date. The corresponding spectrum is plotted in Fig. 3. It is characterized by a FWHM of $50 \mathrm{~nm}$ centered at $1950 \mathrm{~nm}$, supporting a transform-limited pulse duration of $110 \mathrm{fs}$. Note that the intensity envelope is remarkably clean for a broadband fiber amplifier and falls close to a sech ${ }^{2}$ shape. This fact is particularly promising for recompression by use of a suitably designed grating pair and solitonic compression in bulk materials with anomalous group velocity dispersion, which are readily available in this wavelength range. We expect that pulseto-pulse stability of the carrier-envelope phase achieved in our Er:fiber seed source [14] will be preserved throughout the entire system.

In conclusion, we have demonstrated a highly coherent and phase stable seed source for Tm: and Yb:fiber amplifiers. Spectral coherence has been tested in an interferometric experiment comparing two independent amplifier branches and subsequent wavelength conversion in HNFs. We were able to amplify the solitonic portion of the SC in a Tm:fiber amplifier to an energy of $250 \mathrm{~nJ}$. The clean spectral shape of the amplified pulses is promising for further soliton compression in bulk materials. Our system also offers efficient generation of a coherent seed covering the spectral region of Yb:amplifiers around $1 \mu \mathrm{m}$. Therefore, our study paves the way towards spectral combination of mutually coherent Tm: and Yb:fiber amplifiers for the synthesis of single-cycle pulse trains [13]. In the future, the passive phase-locking, together with long wavelength operation at unprecedented average powers and repetition rates, represent key ingredients for compact attosecond technologies.

Support by TOPTICA Photonics AG is gratefully acknowledged. U. Demirbas is grateful for support by the Alexander von Humboldt Foundation.

\section{References}

1. F. Röser, T. Eidam, J. Rothhardt, O. Schmidt, D. N. Schimpf, J. Limpert, and A. Tünnermann, Opt. Lett. 32, 3495 (2007).

2. R. Selm, M. Winterhalder, A. Zumbusch, G. Krauss, T. Hanke, A. Sell, and A. Leitenstorfer, Opt. Lett. 35, 3282 (2010).

3. A. Ruehl, M. J. Martin, K. C. Cossel, L. Chen, H. McKay, B. Thomas, C. Benko, L. Dong, J. Dudley, M. E. Fermann, I. Hartl, and J. Ye, eprint arXiv:1105.2093 (2011).

4. F. Haxsen, D. Wandt, U. Morgner, J. Neumann, and D. Kracht, Opt. Lett. 35, 2991 (2010).

5. R. A. Sims, P. Kadwani, L. Shah, and M. Richardson, in CLEO Conference Digest (2011), paper JWA31.

6. T. Eidam, S. Hanf, E. Seise, T. V. Andersen, T. Wirth, T. Schreiber, J. Limpert, and A. Tünnermann, Opt. Lett. 35, 94 (2010).

7. S. D. Agger and J. H. Povlsen, Opt. Express 14, 50 (2006).

8. G. P. Agrawal, Nonlinear Fiber Optics (Academic Press, 2001).

9. J. Jiang, A. Ruehl, I. Hartl, and M. Fermann, in CLEO/ Europe and EQEC 2011 Conference Digest(2011), paper CF2 4.

10. G. Imeshev and M. Fermann, Opt. Express 13, 7424 (2005).

11. G. Genty, S. Coen, and J. M. Dudley, J. Opt. Soc. Am. B 24, 1771 (2007).

12. T. W. Neely, T. A. Johnson, and S. A. Diddams, Opt. Lett. 36, 4020 (2011).

13. G. Krauss, S. Lohss, T. Hanke, A. Sell, S. Eggert, R. Huber, and A. Leitenstorfer, Nat. Photon. 4, 33 (20010).

14. G. Krauss, D. Fehrenbacher, D. Brida, C. Riek, A. Sell, R. Huber, and A. Leitenstorfer, Opt. Lett. 36, 540 (2011).

15. A. Sell, G. Krauss, R. Scheu, R. Huber, and A. Leitenstorfer, Opt. Express 17, 1070 (2009).

16. F. Tauser and A. Leitenstorfer, "Generation of tunable light pulses, U. S. patent 7,218,443 B2 (May 15, 2007).

17. M. Bellini and T. W. Hänsch, Opt. Lett. 25, 1049 (2000).

18. X. Gu, M. Kimmel, A. Shreenath, R. Trebino, J. Dudley, S. Coen, and R. Windeler, Opt. Express 11, 2697 (2003).

19. J. M. Dudley, G. Genty, and S. Coen, Rev. Mod. Phys. 78, 1135 (2006). 